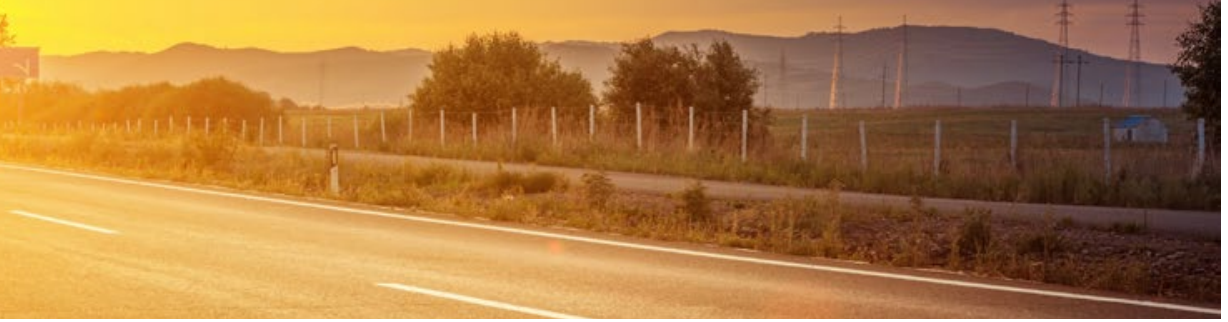

Ergänzungsleistungen - Der gangbare Weg

\title{
Der gesetzlich versicherte Prophylaxepatient
}

\author{
Der gesetzlich Versicherte hat beim Zahnarzt einmal im Jahr den Anspruch auf Entfernung des \\ „Zahnsteins". Diese Leistung wird unter der BEMA-Gebührenposition 107 (Zst) abgerechnet.
}

Der Originalgebührentext der BEMA-Nr. 107 lautet: Entfernen harter Zahnbeläge, je Sitzung. Hierfür gibt es 16 Punkte. Das Entfernen harter Zahnbeläge ist einmal pro Kalenderjahr abrechnungsfähig. Wird ein zweites Mal Zahnstein entfernt, ist dies in jedem Fall privat nach GOZ zu berechnen. Es bietet sich hier an, dem Patienten anstelle einer isolierten Entfernung von Zahnstein (nach den GOZ-Nrn. 4050/4055) eine professionelle Zahnreinigung (nach der GOZ-Nr. 1040) rein unter Prophylaxegesichtspunkten anzubieten. Die meisten Patienten nehmen dieses Angebot verständnisvoll und dankend an.

\section{Definition: Entfernen harter Zahnbeläge}

Da die Bildung von Zahnstein auf der Bildung eines Schmelzoberhäutchens basiert, kann dieser Zahnstein nur am Schmelz lokalisiert sein. Alles, was nicht am Schmelz anhaftet, ist also auch kein Zahnstein. Neben Zahnstein gibt es jedoch auch subgingivale Ablagerungen in Form von Konkrementen am Wurzelzement, deren Entfernung aber nicht mit der Zahnsteinentfernung nach der BEMA-Nr. 107 erfolgt, sondern im Zuge einer systematischen Parodontalbehandlung. Des Weiteren sind dem Lebensalltag geschuldete weiche Beläge dem Zahn aufliegend und Verfärbungen, zum Beispiel aufgrund von Rauchen, Teetrinken und Rotweinkonsum, zu finden. Diese und der Biofilm auf dem Zahngewebe, auf dessen Basis Gingivitiden als auch Parodontitiden entstehen, werden im Zuge einer professionellen Zahnreinigung entfernt.

Eine professionelle Zahnreinigung ist nicht Inhalt des kassenvertraglichen Abrechnungskataloges, sondern findet sich seit 2012 in der privaten Gebührenordnung für Zahnärzte (GOZ).

\section{GOZ-Nr. 1040: Professionelle Zahnreinigung}

Die Leistung umfasst das Entfernen der supragingivalen/gingivalen Beläge auf Zahn- und Wurzeloberflächen einschließlich Reinigung der Zahnzwischenräume, das Entfernen des Biofilms, die Oberflächenpolitur und geeignete Fluoridierungs- maßnahmen, je Zahn oder Implantat oder Brückenglied. Die Leistung nach der Nummer 1040 ist neben den Leistungen nach den Nummern 1020, 4050, 4055, 4060, 4070, 4075, 4090 und 4100 nicht berechnungsfähig.

Sollte man also derartige Maßnahmen nach der GOZ-Nr. 1040 entweder an einem anderen Tag oder an anderen Zähnen oder in einer anderen Sitzung erbringen, so ist dies zusätzlich zur Abrechnung der Bema-Nr. 107 (Zst) möglich. Ausdrücklich möchten wir darauf hinweisen, dass die Kassenzahnärztliche Bundesvereinigung (KZBV) im Schnittstellenpapier zwischen BEMA und GOZ dieses nicht so sieht, weil Behandlungsschritte sich deckten, die in der einen und der anderen Position enthalten seien.

Bei einer ausreichenden Dokumentation der erbrachten Leistungen, das heißt die Behandlungszeit, die Zähne, eine gegebenenfalls nicht sitzungsgleiche Erbringung und den Umfang der Leistungserbringung, können diese Argumente entkräftet werden. Die Wahl eines geeigneten Steigerungsfaktors ist dazu geeignet, den Aufwand der erbrachten Prophylaxeleistung zu differenzieren.

In der nächsten Ausgabe werden wir uns mit der Kassenkrone auseinandersetzen und wieder Ergänzungsleistungen und den hierbei gangbaren Weg aufzeigen.

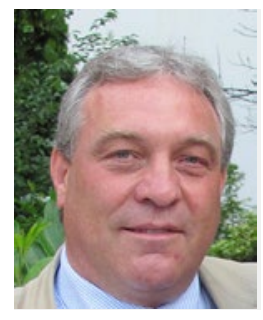

Dr. Christian Öttl

Mitglied im FVDZ-Bundesvorstand 